\title{
Positivity of Fundamental Matrix and Exponential Stability of Delay Differential System
}

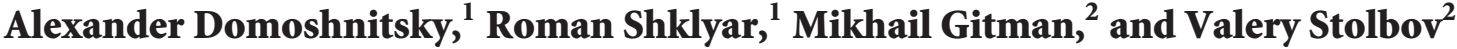 \\ ${ }^{1}$ Department of Mathematics and Computer Sciences, Ariel University, Ariel, Israel \\ ${ }^{2}$ Department of Mathematical Modelling, Perm National Research Polytechnic University, Perm, Russia
}

Correspondence should be addressed to Alexander Domoshnitsky; adom@ariel.ac.il

Received 1 March 2014; Accepted 22 July 2014; Published 28 August 2014

Academic Editor: Yuriy Rogovchenko

Copyright (c) 2014 Alexander Domoshnitsky et al. This is an open access article distributed under the Creative Commons Attribution License, which permits unrestricted use, distribution, and reproduction in any medium, provided the original work is properly cited.

\begin{abstract}
The classical Wazewski theorem established that nonpositivity of all nondiagonal elements $p_{i j}(i \neq j, i, j=1, \ldots, n)$ is necessary and sufficient for nonnegativity of the fundamental (Cauchy) matrix and consequently for applicability of the Chaplygin approach of approximate integration for system of linear ordinary differential equations $x_{i}^{\prime}(t)+\sum_{j=1}^{n} p_{i j}(t) x_{j}(t)=f_{i}(t), i=1, \ldots, n$. Results on nonnegativity of the Cauchy matrix for system of delay differential equations $x_{i}^{\prime}(t)+\sum_{j=1}^{n} p_{i j}(t) x_{j}\left(h_{i j}(t)\right)=f_{i}(t), i=$ $1, \ldots, n$, which were based on nonpositivity of all diagonal elements, were presented in the previous works. Then examples, which demonstrated that nonpositivity of nondiagonal coefficients $p_{i j}$ is not necessary for systems of delay equations, were found. In this paper first sufficient results about nonnegativity of the Cauchy matrix of the delay system without this assumption are proven. A necessary condition of nonnegativity of the Cauchy matrix is proposed. On the basis of these results on nonnegativity of the Cauchy matrix, necessary and sufficient conditions of the exponential stability of the delay system are obtained.
\end{abstract}

\section{Introduction}

Consider the system

$$
\begin{gathered}
\left(M_{i} x\right)(t) \equiv x_{i}^{\prime}(t)+\sum_{j=1}^{n} p_{i j}(t) x_{j}\left(h_{i j}(t)\right)=f_{i}(t), \\
i=1, \ldots, n, \quad t \in[0, \infty), \\
x_{i}(\theta)=0 \quad \text { for } \theta<0, i=1, \ldots, n,
\end{gathered}
$$

where $p_{i j}$ are measurable essentially bounded functions and $h_{i j}$ are measurable functions such that $h_{i j}(t) \leq t$ for $i, j=$ $1, \ldots, n, t \in[0, \infty)$.

Tchaplygin [1] proposed the method of approximate integration which was based on the following fact: from the inequalities

$$
\begin{array}{r}
\left(M_{i} x\right)(t) \geq\left(M_{i} y\right)(t), \\
t \in[0, \infty), \quad x_{i}(0) \geq y_{i}(0), \quad i=1, \ldots, n,
\end{array}
$$

it follows that

$$
x_{i}(t) \geq y_{i}(t), \quad t \in[0, \infty), i=1, \ldots, n .
$$

Many works, started with the known paper by Luzin [2], were devoted to the various aspects of Tchaplygin's approximate method of integration. The well-known monograph [3] opened the series of books developing monotone methods which were based on this property. Note the monograph [4], where the monotone technique was used for analysis of existence, uniqueness, and estimates of solutions to various boundary value problems for systems of functional differential equations.

The general solution of system (1) with the initial functions (2) can be represented in the following form:

$$
x(t)=\int_{0}^{t} C(t, s) f(s) d s+C(t, 0) x(0)
$$

where $f(t)=\operatorname{col}\left\{f_{1}(t), \ldots, f_{n}(t)\right\}, \quad x(0)=$ $\operatorname{col}\left\{x_{1}(0), \ldots, x_{n}(0)\right\}$, and $C(t, s)=\left\{C_{i j}(t, s)\right\}_{i, j=1}^{n}$ is called the Cauchy matrix of system (1). Note that, for every fixed $s$, the matrix $C(t, s)$ is the fundamental matrix of system (1) such that $C(s, s)=I$ ( $I$ is the unit $n \times n$ matrix) [5]; that is, the $j$ th column $\operatorname{col}\left\{C_{1 j}(t, s), \ldots, C_{n j}(t, s)\right\}$ of the Cauchy matrix 
$C(t, s)$ for every fixed $s$ as a function of $t$ is the solution of the system

$$
\begin{array}{r}
x_{i}^{\prime}(t)+\sum_{j=1}^{n} p_{i j}(t) x_{j}\left(h_{i j}(t)\right)=0, \\
i=1, \ldots, n, \quad t \in[s, \infty), \\
x_{i}(\theta)=0 \quad \text { for } \theta<s, i=1, \ldots, n,
\end{array}
$$

satisfying the initial condition $\operatorname{col}\left\{C_{1 j}(s, s), \ldots, C_{n j}(s, s)\right\}=$ $\operatorname{col}\left\{\delta_{1 j}, \ldots, \delta_{n j}\right\}$, where $\delta_{j j}=1$ and $\delta_{i j}=0$ for $i \neq j$. Nonnegativity of all entries $C_{i j}(t, s)$ of the Cauchy matrix $C(t, s)$ is equivalent to the property $(3) \Rightarrow(4)$.

As a particular case of (1), the system of ordinary differential equations

$$
x_{i}^{\prime}(t)+\sum_{j=1}^{n} p_{i j}(t) x_{j}(t)=f_{i}(t), \quad i=1, \ldots, n, t \in[0, \infty),
$$

can be considered.

The classical Wazewski theorem claims [6] that the condition

$$
p_{i j} \leq 0 \text { for } j \neq i, i, j=1, \ldots, n,
$$

is necessary and sufficient for nonnegativity of all elements $C_{i j}(t, s)$ of the Cauchy matrix and consequently of the property $(3) \Rightarrow(4)$ for system of ordinary differential equations (7) with continuous coefficients. Sufficient conditions of nonnegativity of all elements of the Cauchy matrix $C(t, s)$ for system of delay equations (1) were first obtained and used for the study of the exponential stability in the paper [7].

It should be noted here that the classical monotone technique in the theory of boundary value problems for nonlinear differential systems is based on monotonicity of corresponding operators acting on spaces of corresponding vector-functions; that is, the operators act in corresponding cones. These operators are obtained as a result of regularization procedures reducing the boundary value problems to equivalent integral equations $[3-5,8,9]$. The monotonicity of such operators is obtained on the basis of positivity of entries of Cauchy or Green's matrices of corresponding boundary value problems.

The problem of the exponential stability of delay differential systems is one of the most important applications of results on positivity of the Cauchy matrix $C(t, s)$.

Definition 1. One says that the Cauchy matrix of system (1) satisfies the exponential estimate if there exist constants $N$ and $\alpha$ such that

$$
\begin{aligned}
& \quad\left|C_{i j}(t, s)\right| \leq N \exp (-\alpha(t-s)) \\
& \text { for } 0 \leq s \leq t<\infty, \quad i, j=1, \ldots, n .
\end{aligned}
$$

It is known [5] that in the case of bounded delays $t-h_{i j}(t)$ $(i, j=1, \ldots, n)$ the exponential estimate of the Cauchy matrix $C(t, s)$ is equivalent to the uniform exponential stability.

The technique of the use of positivity of $C(t, s)$ in the exponential stability was proposed in the papers $[7,10]$, where necessary and sufficient conditions of the exponential stability for systems, possessing positivity of the Cauchy matrix, were obtained.

Theorem A (see [7]). Let all delays $t-h_{i j}(t)$ be bounded and let the coefficients $p_{i j}$ be constants, $p_{i j} \leq 0$ for $i \neq j, p_{i i}(t-$ $\left.h_{i i}(t)\right) \leq 1 / e$, for $i, j=1, \ldots, n, t \in[0, \infty)$. Then the following two conditions (a) and (b) are equivalent.

(a) The Cauchy matrix of system (1) satisfies the exponential estimate (9).

(b) All the components of the unique solution $z=$ $\operatorname{col}\left\{z_{1}, \ldots, z_{n}\right\}$ of the linear algebraic system

$$
\sum_{j=1}^{n} p_{i j} z_{j}=1, \quad i=1, \ldots, n,
$$

are positive.

For the case of variable coefficients $p_{i j}(t)$ and without the assumption $p_{i j}(t) \leq 0$ for $i \neq j, i, j=1, \ldots, n, t \in[0, \infty)$, the following assertion was obtained.

Theorem B (see [10]). Assume that the following conditions (1)-(3) are fulfilled.

(1) All the delays $t-h_{i j}(t)$ are bounded for $t \in[0, \infty)$, $i, j=1, \ldots, n$.

(2) The inequalities

$$
\int_{h_{i j}(t)}^{t} p_{i i}(s) d s \leq \frac{1}{e}, \quad t \in[0, \infty), i=1, \ldots, n,
$$

where $p_{i i}(s) \equiv 0$ for $s<0$, are true.

(3) There exist positive $z_{1}, \ldots, z_{n}$ and $\varepsilon$ such that

$$
p_{i i}(t) z_{i}-\sum_{j=1, j \neq i}^{n}\left|p_{i j}(t)\right| z_{j} \geq \varepsilon>0, \quad i=1, \ldots, n .
$$

Then the Cauchy matrix of system (1) satisfies the exponential estimate (9).

Remark 2 (see [10]). If we choose $z_{1}=1, \ldots, z_{n}=1$, in condition (3), we obtain the following simple inequalities:

$$
p_{i i}(t)-\sum_{j=1, j \neq i}^{n}\left|p_{i j}(t)\right| \geq \varepsilon>0, \quad i=1, \ldots, n .
$$

In other terminologies reducing a delay system to an equivalent system of integral equations and estimating its norm can be found in the paper [11]. Then the idea of a regularization, leading to analysis of vector integral equations with positive operators, was proposed in the papers [12,13]. A development of this approach can be found in $[14,15]$. In all results of this sort we can find an assumption about dominant main diagonal.

It seems that condition (8) is necessary also for delay system (1). That is why the following development of Tchaplygin's idea was presented in the paper [16]. 
Let $k_{i}$ be either 1 or 2 . In [16] the following problem is formulated: when from the conditions

$$
\begin{array}{r}
(-1)^{k_{i}}\left[\left(M_{i} x\right)(t)-\left(M_{i} y\right)(t)\right] \geq 0, \\
t \in[0, \infty), \quad x_{i}(0)=y_{i}(0), \quad i=1, \ldots, n,
\end{array}
$$

it does follow that for a corresponding fixed $r$ the component $x_{r}$ of the solution vector the inequality

$$
x_{r}(t) \geq y_{r}(t), \quad t \in[0, \infty),
$$

is satisfied. This property is a weakening of property (3) $\Rightarrow$ (4) and leads to essentially less hard than condition (8) limitations on the given system. From the formula of solution's representation it follows that this property is reduced to signconstancy of all elements standing only in the $r$ th row of the Cauchy matrix. This idea was developed in $[14,17]$ and used for the study of the exponential stability in $[16,18]$ without assumption about the dominant main diagonal.

Example, where one of the nondiagonal coefficients $p_{i_{0} j_{0}}>0$ but all entries of the Cauchy matrix are nonnegative, was constructed first in [17] on the finite interval and in [19] on semiaxis. The cases when (8) is a necessary condition for nonnegativity of all entries of the Cauchy matrix were discussed in [19].

In the second paragraph of this paper we obtain sufficient conditions of nonnegativity of all entries of the Cauchy matrix in the case of existence of nondiagonal positive coefficient $p_{i_{0} j_{0}}$ and obtain new necessary condition for this nonnegativity. On this basis, we obtain necessary and sufficient conditions of the exponential stability in Section 3, which generalize Theorems A and B in this case. In Section 4, various examples are proposed. They demonstrate that obtained results allow us to make conclusions about nonnegativity of the Cauchy matrix and the exponential stability in cases for which the results of $[7,10,14,17,19]$ cannot be used.

\section{Positivity of the Cauchy Matrix in the Case of One Positive Nondiagonal Coefficient}

As usual we say that an operator $A: X \rightarrow Y$ is positive if for every nonnegative $f \in X$ we get nonnegative $A f \in Y$ ( $X$ and $Y$ are corresponding function spaces). Nonnegativity of vector functions $f=\operatorname{col}\left\{f_{1}, \ldots, f_{n}\right\}$ we understand as nonnegativity of all the components $f_{1}, \ldots, f_{n}$. Before formulations of the main assertions of this paragraph, note that positivity of the Cauchy operator

$$
(C f)(t)=\int_{0}^{t} C(t, s) f(s) d s
$$

(see formula of solution's representation (5)) follows from nonnegativity of all entries of the Cauchy matrix $C(t, s)=$ $\left\{C_{i j}(t, s)\right\}_{i, j=1}^{n}$. We say that the Cauchy matrix $C(t, s)$ is not positive if there exist a point $\left(t_{0}, s_{0}\right)$ and an entry $C_{i_{0} j_{0}}(t, s)$, such that $C_{i_{0} j_{0}}\left(t_{0}, s_{0}\right)<0$. The Cauchy operator $C$ is not positive, if there exist points $\left(t_{0}, s\right)$ such that mes $\left\{s: C\left(t_{0}, s\right)<\right.$ $0\}>0$.
Consider the following auxiliary system:

$$
\begin{array}{r}
x_{i}^{\prime}(t)+\sum_{j=1}^{n} q_{i j}(t) x_{j}\left(h_{i j}(t)\right)=z_{i}(t), \\
i=1, \ldots, n, \quad t \in[0, \infty), \\
x_{i}(\theta)=0 \quad \text { for } \theta<0, i=1, \ldots, n,
\end{array}
$$

where

$$
q_{i j}(t)=\left\{\begin{array}{lr}
p_{i j}(t), & i \neq 1, j=1, \ldots, n \\
p_{i j}(t), & i=1, j=1, \ldots, n-2, \\
0, & i=1, j=n-1, j=n \\
i=1, \ldots, n .
\end{array}\right.
$$

Denote by $W(t, s)=\left\{W_{i j}(t, s)\right\}_{i, j=1}^{n}$ the Cauchy matrix of system (17).

Theorem 3. Let the conditions (1), (2), and (3) be fulfilled:

(1) $p_{i j} \leq 0$ for $i \neq j,(i, j) \neq(1, n), i, j=1, \ldots, n$,

(2) condition (2) of Theorem $B$ is true,

(3) the inequalities

$$
\begin{array}{r}
p_{1 n}(t) W_{n j}\left(h_{1 n}(t), s\right) \chi\left(h_{1 n}(t), s\right) \\
+p_{1 n-1}(t) W_{n-1, j}\left(h_{1 n-1}(t), s\right) \chi\left(h_{1 n-1}(t), s\right) \leq 0, \\
j=1, \ldots, n,
\end{array}
$$

where $0 \leq s \leq t<\infty$ and

$$
\chi\left(h_{1 k}(t), s\right)=\left\{\begin{array}{ll}
1, & \text { for } h_{1 k}(t) \geq s \\
0, & \text { for } h_{1 k}(t)<s
\end{array},\right.
$$

for every $k=1, \ldots, n$, are true.

Then the Cauchy matrix $C(t, s)$ of system (1) is nonnegative for $0 \leq s \leq t<\infty$.

Remark 4. Instead of inequality (11) in condition (2) of Theorem 3 we can require that the Cauchy functions $c_{i}(t, s)$ of $n$ scalar first order diagonal equations

$$
x_{i}^{\prime}(t)+p_{i i}(t) x_{j}\left(h_{i i}(t)\right)=0, \quad t \in[0, \infty), i=1, \ldots, n,
$$

with initial functions defined by (18) are positive. Inequalities (11) imply [20] that $c_{i}(t, s)>0$ for $0 \leq s \leq t<\infty$.

Proof. Let us use the transform $x=W z$, where $W$ is the Cauchy operator of system (17), which is defined by the equality

$$
(W z)(t)=\int_{0}^{t} W(t, s) z(s) d s,
$$

with the Cauchy matrix $W(t, s)=\left\{W_{i j}(t, s)\right\}_{i, j=1}^{n}$ of system (17). We obtain

$$
z(t)-(K z)(t)=f(t),
$$


where the operator $K: L_{\infty} \rightarrow L_{\infty}$ is defined by the equality

$(K z)(t)$

$$
\begin{aligned}
=-\operatorname{col}\{ & p_{1 n}(t) \chi\left(h_{1 n}(t), 0\right) \\
& \times \sum_{j=1}^{n} \int_{0}^{h_{1 n}(t)} W_{n j}\left(h_{1 n}(t), s\right) z_{j}(s) d s \\
& +p_{1 n-1}(t) \chi\left(h_{1 n-1}(t), 0\right) \\
& \left.\times \sum_{j=1}^{n} \int_{0}^{h_{1 n-1}(t)} W_{n-1, j}\left(h_{1 n-1}(t), s\right) z_{j}(s) d s, 0, \ldots, 0\right\},
\end{aligned}
$$

where $W(t, s)=0$ for $t<s$.

Condition (3) in Theorem 3 implies that the operator $K: L_{\infty} \rightarrow L_{\infty}$ defined by equality (25) is positive. On every finite interval $[0, \omega]$ the spectral radius of this operator $K$ is equal to zero [5]. Then the operator $(I-K)^{-1}$ is positive and all components of the solution-vector $z=(I-$ $K)^{-1} f$ are nonnegative for every vector $f$ with nonnegative components. We have proven that the Cauchy operator $C$ of system (1) defined by the equalities

$$
(C f)(t) \equiv \int_{0}^{t} C(t, s) f(s) d s=\int_{0}^{t} W(t, s)(I-K)^{-1} f(s) d s,
$$

where $C(t, s)=\left\{C_{i j}(t, s)\right\}_{i, j=1}^{n}$ is the Cauchy matrix of system (1), is positive. It follows from (26) and nonnegativity of the Cauchy matrix $W(t, s)$ of system (17) that $C(t, s) \geq W(t, s)$ and consequently all entries $C_{i j}(t, s)$ of the Cauchy matrix $C(t, s)$ are nonnegative.

Remark 5. Note that, in the case $p_{1 n} \geq 0$ and $\operatorname{essup}_{t \geq 0} p_{1 n}(t)>0$, we actually require in Theorem 3 that $h_{1 n}(t)<h_{1 n-1}(t)$ to obtain positivity of the Cauchy matrix $C(t, s)$. This inequality on delays is essential. Theorem 6 demonstrates that, in the case of the opposite inequality $h_{1 n}(t) \geq h_{1 n-1}(t)$, condition (8) is actually a necessary one.

Assume that $h_{i j}(t)$ is a nondecreasing function $\left(h_{i j}(t) \leq t\right)$ and define $t_{s}^{*}$ as an exact upper limit of the set of such $t \geq s$ for which $h_{i j}(t) \leq s$.

Theorem 6. Let the following conditions be fulfilled:

(1) the Cauchy functions $c_{i}(t, s)$ of all diagonal equations (22) with the initial functions defined by (18) are positive for $0 \leq s \leq t<\infty$;

(2) $h_{k j}(t)$ are nondecreasing functions for $k, j=1, \ldots, n$;

(3) there exist an interval $\left[t_{0}, t_{1}\right]$, a coefficient $p_{i_{0} j_{0}}$ with $i_{0} \neq j_{0}$, and a positive constant $\delta$ such that $t_{t_{0}}^{*}<$ $t_{1}, p_{i_{0} j_{0}}(t) \geq \delta$, and $h_{i_{0} j_{0}}(t) \geq h_{k j}(t)$, for all $k \neq j$, $k, j=1, \ldots, n$, in the interval $t \in\left[t_{0}, t_{1}\right]$.
Then,

(a) the fundamental matrix $C(t, s)$ of system (1) cannot be nonnegative in the triangle $0 \leq s \leq t<\infty$;

(b) if mes $\left\{s: t_{0} \leq s \leq t_{s}^{*}<t_{1}\right\}>0$, then the Cauchy operator $(C f)(t) \equiv \int_{0}^{t} C(t, s) f(s) d s$ cannot be positive.

Proof. Without loss of generality we can assume that $i_{0}=$ $n, j_{0}=1$; that is, $p_{n 1}(t) \geq \delta>0$ in $\left[t_{0}, t_{1}\right]$. Consider now the first column $X(t)=\operatorname{col}\left\{C_{i 1}(t, s)\right\}_{i=1}^{n}$ of the Cauchy matrix $C(t, s)$. This column $X(t)$ is the solution of system $(6)$, satisfying the condition $X(s)=\operatorname{col}\{1,0, \ldots, 0\}$. We assume that $t_{0} \leq s<t_{1}$ such that $t_{s}^{*}<t_{1}$. Consider now the $n$th component $X_{n}(t)$ of this solution-vector. It satisfies the following:

$$
\begin{gathered}
X_{n}^{\prime}(t)+p_{n n}(t) X_{n}\left(h_{n n}(t)\right)=-\sum_{j=1, j \neq n}^{n} p_{n j}(t) X_{j}\left(h_{n j}(t)\right), \\
X_{n}(\theta)=0 \quad \text { for } \theta<s,
\end{gathered}
$$

and the condition $X_{n}(s)=0$. According to condition (3) in Theorem 3, we have $h_{n 1}(t) \geq s$ for $t \geq t_{s}^{*}$. It is clear that $X_{1}(t) \equiv 1, X_{j}(t) \equiv 0$ for $j=2, \ldots, n, t \in\left[s, t_{s}^{*}\right]$ and there exists an interval $\left[t_{s}^{*}, t_{s}^{*}+\varepsilon\right]$ such that

$$
\begin{array}{r}
\psi(t) \equiv-\sum_{j=1, j \neq n}^{n} p_{n j}(t) X_{j}\left(h_{n j}(t)\right)<0, \\
\quad i=1, \ldots n, \quad t \in\left(t_{s}^{*}, t_{s}^{*}+\varepsilon\right] .
\end{array}
$$

The representation of solution of scalar equation (27) with the initial condition $X_{n}(s)=0$ leads us to

$$
X_{n}(t)=\int_{s}^{t} c_{n}(t, s) \psi(s) d s<0, \quad t \in\left(t_{s}^{*}, t_{s}^{*}+\varepsilon\right] .
$$

Thus we obtain that $X_{n}(t)=C_{n 1}(t, s)<0$ for $t \in\left(t_{s}^{*}, t_{s}^{*}+\varepsilon\right]$.

This completes the proof of Theorem 6 .

Example 7. Consider the following system with one constant delay:

$$
\begin{array}{r}
x_{i}^{\prime}(t)+\sum_{j=1}^{n} p_{i j}(t) x_{j}(t-\tau)=f_{i}(t), \\
i=1, \ldots, n, \quad t \in[0, \infty), \\
x_{i}(\xi)=0, \quad \text { for } \xi<0, i=1, \ldots, n .
\end{array}
$$

If $p_{i_{0} j_{0}}(t) \geq \delta>0$ for $i_{0} \neq j_{0}, t \in\left[t_{0}, t_{1}\right]$, such that $\tau<$ $t_{1}-t_{0}$, then, under the conditions of Theorem $6, C(t, s)$ cannot be nonnegative.

In the case of constant coefficients $p_{i j}(t)=p_{i j}$ and under the condition $p_{i i} \tau \leq 1 / e$ for all $i=1, \ldots, n$, implying positivity of the Cauchy functions $c_{i}(t, s)$ of diagonal equations (22), and the assumption that $h_{k j}$ are nondecreasing for $k, j=$ $1, \ldots, n$, the condition $p_{i j} \leq 0$ for all $i \neq j, i, j=1, \ldots, n$, is necessary and sufficient for nonnegativity of $C(t, s)$.

Let us demonstrate that the assertion (a) of Theorem 6 can be true, but the assertion (b) is not true. 
Example 8. Consider the following system:

$$
\begin{gathered}
x_{i}^{\prime}(t)=0, \quad i=1, \ldots, n-1, \\
x_{n}^{\prime}(t)+p_{n 1} x_{1}(0)=0, \quad t \in[0, \infty) .
\end{gathered}
$$

For every $s>0$ we have $C(t, s)=I$, where $I$ is the unit $n \times n$ matrix. For $s=0$, we have $C_{n 1}(t, 0)=-p_{n 1} t<0$, but the operator $(C f)(t) \equiv \int_{0}^{t} C(t, s) f(s) d s$ does not "feel" this and is positive.

\section{About Exponential Stability of Delay Systems}

Now let us study the exponential stability of system (1) on the basis of positivity of its Cauchy matrix $C(t, s)$.

Let us assume that $p_{1 n}(t) \geq 0, p_{1 n-1}(t) \leq 0$ and extend the coefficients and delays on the interval $[-H, 0)$, where $H=\max _{i, j=1, \ldots, n} \operatorname{esssup}_{t \geq 0}\left\{t-h_{i j}(t)\right\}$ as follows: $h_{i j}(t) \equiv t$ for $t \in[-H, 0], i, j=1, \ldots, n, p_{11}(t)=\operatorname{essup}_{t \geq 0}\left\{p_{11}(t)+\right.$ $\left.p_{1 n}(t)\right\}, p_{i i}(t)=\operatorname{esssup}_{t \geq 0} p_{i i}(t)$ for $i=2, \ldots, n, p_{i j}(t) \equiv 0$ for $i \neq j, t \in-[H, 0]$.

Consider the following auxiliary system:

$$
\begin{array}{r}
x_{i}^{\prime}(t)+\sum_{j=1}^{n} p_{i j}(t) x_{j}\left(h_{i j}(t)\right)=f_{i}(t), \\
\quad i=1, \ldots, n, \quad t \in[-H, \infty),
\end{array}
$$

where $f_{i}$ are measurable essentially bounded functions $f_{i}$ : $[-H, \infty) \rightarrow(-\infty,+\infty), i=1, \ldots, n$.

Theorem 9. Let $p_{1 n}(t) \geq 0, p_{1 n-1}(t) \leq 0$, let all conditions of Theorem 3 be fulfilled on $[-H, \infty)$, let all delays $t-$ $h_{i j}(t)$ be bounded, and let there exist a constant-vector $z=$ col $\left\{z_{1}, \ldots, z_{n}\right\}$ with all positive components $z_{1}, \ldots, z_{n}$ such that

$$
\sum_{j=1}^{n} p_{i j}(t) z_{j} \geq \varepsilon>0, \quad i=1, \ldots, n .
$$

Then the Cauchy matrix $C(t, s)$ of system (1) satisfies the exponential estimate (9). If $\varepsilon \geq 1$, then the following estimate is true:

$$
\int_{0}^{t} \sum_{j=1}^{n} C_{i j}(t, s) d s \leq z_{i}, \quad i=1, \ldots, n .
$$

Proof. The vector-function $z=\operatorname{col}\left\{z_{1}, \ldots, z_{n}\right\}$ satisfies the following system:

$$
\begin{array}{r}
x_{i}^{\prime}(t)+\sum_{j=1}^{n} p_{i j}(t) x_{j}\left(h_{i j}(t)\right) \equiv g_{i}(t) \geq \varepsilon, \\
i=1, \ldots, n, \quad t \in-[H, \infty),
\end{array}
$$

and the initial conditions $x_{i}(-H)=z_{i}, i=1, \ldots, n$. The solution representation formula (5) allows us to write for this initial value problem

$$
z=\int_{-H}^{t} C(t, s) g(s) d s+C(t,-H) z
$$

where $g(t)=\operatorname{col}\left\{g_{1}(t), \ldots, g_{n}(t)\right\}$. Theorem 3 implies nonnegativity of all entries $C_{i j}(t, s)$ of the Cauchy matrix. From nonnegativity of all entries of the Cauchy matrix $C(t, s)$ and all components of the vector $z=\operatorname{col}\left\{z_{1}, \ldots, z_{n}\right\}$, it follows that, for every bounded right hand side $f=\operatorname{col}\left\{f_{1}, \ldots, f_{n}\right\}$, the solution $x=\operatorname{col}\left\{x_{1}, \ldots, x_{n}\right\}$ is bounded. From this fact and Theorem 3.4 of paragraph 5.3 of [5] the exponential estimate (9) of the Cauchy matrix follows. The Cauchy matrices of system (1) and (32) coincide for $0 \leq s \leq t<\infty$.

The positivity of $C(t, s)$ and $z$ and (36) imply the inequality (34).

Theorem 10. Let all coefficients $p_{i j}$ be constants, let all delays $t-h_{i j}(t)$ be bounded $(i, j=1, \ldots, n)$, and let conditions of Theorem 3 be fulfilled on $[-H, \infty)$. Then the Cauchy matrix $C(t, s)$ of system (1) satisfies the exponential estimate if and only if all components $z_{1}, \ldots, z_{n}$ of the solution $z=\operatorname{col}\left\{z_{1}, \ldots, z_{n}\right\}$ of the algebraic system

$$
\sum_{j=1}^{n} p_{i j} z_{j}=1, \quad i=1, \ldots, n
$$

are positive.

Proof. The sufficiency follows from Theorem 9. To prove necessity we note that the constant vector $z=\operatorname{col}\left\{z_{1}, \ldots, z_{n}\right\}$ is the solution of the following system:

$$
\begin{array}{r}
x_{i}^{\prime}(t)+\sum_{j=1}^{n} p_{i j}(t) x_{j}\left(h_{i j}(t)\right)=1, \\
i=1, \ldots, n, \quad t \in-[H, \infty),
\end{array}
$$

satisfying the condition $x_{i}(-H)=z_{i}, i=1, \ldots, n$. Writing the representation of its solution, we get

$$
z_{i}=\int_{-H}^{t} \sum_{j=1}^{n} C_{i j}(t, s) d s+\sum_{j=1}^{n} C_{i j}(t,-H) z_{j}, \quad i=1, \ldots, n .
$$

All entries of the matrix $C(t, s)$ are nonnegative, according to Theorem 3 , and $C_{i i}(t, s)>0$ for $0 \leq s \leq t<\infty$ according to the results of paragraph 16 of [14]. Now from the exponential estimate of the Cauchy matrix, it follows that all components $z_{i}$ are positive for $i=1, \ldots, n$.

Remark 11. In the case of nonnegativity and the exponential estimate of the Cauchy matrix $C(t, s)$, it follows that

$$
\lim _{t \rightarrow \infty} \int_{0}^{t} \sum_{j=1}^{n} C_{i j}(t, s) d s=z_{i}, \quad i=1, \ldots, n .
$$

Consider the following system:

$$
\begin{array}{r}
x_{i}^{\prime}(t)+\sum_{j=1}^{n} p_{i j}(t) x_{j}\left(h_{i j}(t)\right)+\sum_{j=1}^{n} b_{i j}(t) x_{j}\left(g_{i j}(t)\right) \equiv f_{i}(t), \\
i=1, \ldots, n, \quad t \in[0, \infty) .
\end{array}
$$


where $g_{i j}$ are measurable functions, $g_{i j}(t) \leq t, b_{i j}$ are measurable essentially bounded functions for $i, j=1, \ldots, n$.

Theorem 12. Let for system (1) all conditions of Theorem 9 be fulfilled, and let all delays $t-g_{i j}(t), t-h_{i j}(t)$ for $i, j=1, \ldots, n$ be bounded and

$$
\max \left\{z_{1}, \ldots, z_{n}\right\} \underset{t \geq 0}{\operatorname{esssup}} \sum_{i=1}^{n} \sum_{j=1}^{n}\left|b_{i j}(t)\right|<1,
$$

Then the Cauchy matrix $C(t, s)$ of system (41) satisfies the exponential estimate (9).

Proof. Theorem 3 implies that the Cauchy matrix $W(t, s)$ of system (1) is nonnegative. Theorem 9 implies that $W(t, s)$ satisfies the exponential estimate and its entries $W_{i j}(t, s)$ satisfy the following inequalities:

$$
\int_{0}^{t} \sum_{j=1}^{n} W_{i j}(t, s) d s \leq z_{i}, \quad i=1, \ldots, n .
$$

We can write system (41) in the following equivalent form:

$$
x(t)=(K x)(t)+W(t, 0) x(0)+\psi(t),
$$

where the operator $K: C \rightarrow C$ ( $C$ is the space of continuous $n$-vector-functions) is defined by the following equality:

$(K x)(t)$

$$
\begin{aligned}
& =-\int_{0}^{t} W(t, s) \operatorname{col}\left\{\sum_{j=1}^{n} b_{1 j}(s) x_{j}\left(g_{1 j}(s)\right) d s, \ldots,\right. \\
& \left.\qquad \sum_{j=1}^{n} b_{n j}(s) x_{j}\left(g_{n j}(s)\right) d s\right\}, \\
& \psi(t)=\int_{0}^{t} W(t, s) \operatorname{col}\left\{f_{1}(s), \ldots, f_{n}(s)\right\} d s .
\end{aligned}
$$

The exponential estimate of the Cauchy matrix $W(t, s)$ implies that $W(t, 0) x(0)+\psi(t)$ is bounded. Condition (42) implies the estimate of the norm $\|K\|<1$. Thus for every bounded right hand side the solution $x$ of system (41) is bounded. This, according to Theorem 3.4 [5], implies the exponential estimate (9) of the Cauchy matrix $C(t, s)$ of system (41).

\section{Examples}

Example 13. Let us consider the following delay system:

$$
\begin{gathered}
x_{1}^{\prime}(t)+x_{1}(t)-x_{2}(t)+x_{3}(t-10)=f_{1}(t), \\
x_{2}^{\prime}(t)-x_{1}(t)+3 x_{2}(t)-x_{3}(t)=f_{2}(t) \\
x_{3}^{\prime}(t)-x_{1}(t)-x_{2}(t)+3 x_{3}(t)=f_{3}(t) .
\end{gathered}
$$

The Wazewski condition (8) about nonpositivity of $p_{i j}$ for $i \neq j$ is not fulfilled since $p_{13}(t) \equiv 1$ for $t \in[0, \infty)$.
Consequently Theorem A does not work here. Conditions (1) and (2) of Theorem B are fulfilled, but (3) is not true. Of course, condition (13) is not fulfilled. We cannot make conclusions about nonnegativity of the Cauchy matrix $C(t, s)$ and its exponential estimate based on Theorems A and B and all other previous results. Let us use the results obtained in our paper.

Consider the following auxiliary system:

$$
\begin{gathered}
x_{1}^{\prime}(t)+x_{1}(t)=z_{1}(t), \\
x_{2}^{\prime}(t)-x_{1}(t)+3 x_{2}(t)-x_{3}(t)=z_{2}(t), \\
x_{3}^{\prime}(t)-x_{1}(t)-x_{2}(t)+3 x_{3}(t)=z_{3}(t) .
\end{gathered}
$$

The Cauchy matrix of this auxiliary system is denoted by $W(t, s)=\left\{W_{i j}(t, s)\right\}_{i, j=1,2,3}$. It can be found that

$W(t, s)=\left[\begin{array}{ccc}e^{(s-t)} & 0 & 0 \\ e^{(s-t)}-e^{2(s-t)} & \frac{e^{2(s-t)}+e^{4(s-t)}}{2} & \frac{e^{2(s-t)}-e^{4(s-t)}}{2} \\ e^{(s-t)}-e^{2(s-t)} & \frac{e^{2(s-t)}-e^{4(s-t)}}{2} & \frac{e^{2(s-t)}+e^{4(s-t)}}{2}\end{array}\right]$

We see that condition (20) is,

$$
\begin{aligned}
& p_{13} W_{31}(t-10, s)-p_{12} W_{21}(t, s) \\
& =\left(e^{(s-t+10)}-e^{2(s-t+10)}\right)-\left(e^{(s-t)}-e^{2(s-t)}\right)<0, \\
& p_{13} W_{32}(t-10, s)-p_{12} W_{22}(t, s) \\
& =\left(\frac{e^{2(s-t+10)}-e^{4(s-t+10)}}{2}\right)-\left(\frac{e^{2(s-t)}+e^{4(s-t)}}{2}\right)<0,
\end{aligned}
$$

true. According to Theorem 3, all elements of the Cauchy matrix $C(t, s)=\left\{C_{i j}(t, s)\right\}_{i, j=1,2,3}$ are nonnegative for $0 \leq s \leq$ $t<\infty$. System (37) in this case is of the following form:

$$
\begin{gathered}
z_{1}-z_{2}+z_{3}=1, \\
-z_{1}+3 z_{2}-z_{3}=1, \\
-z_{1}-z_{2}+3 z_{3}=1 .
\end{gathered}
$$

Positivity of all components of its solution is necessary and sufficient for the exponential estimate (9) of the Cauchy matrix $C(t, s)$ of the delay system $(46)$ according to Theorem 10. The solution of the algebraic system (50) is the following: $z_{1}=1, z_{2}=1$, and $z_{3}=1$. This implies the 
exponential estimate of the Cauchy matrix $C(t, s)$ of the delay system. We get also (see Remark 11) that

$$
\begin{aligned}
& z_{1}=1=\lim _{t \rightarrow \infty} \sum_{j=1}^{n} \int_{0}^{t} C_{1 j}(t, s) d s, \\
& z_{2}=1=\lim _{t \rightarrow \infty} \sum_{j=1}^{n} \int_{0}^{t} C_{2 j}(t, s) d s, \\
& z_{3}=1=\lim _{t \rightarrow \infty} \sum_{j=1}^{n} \int_{0}^{t} C_{3 j}(t, s) d s .
\end{aligned}
$$

Example 14. The same comments can be done also for the delay system of 4 equations:

$$
\begin{gathered}
x_{1}^{\prime}(t)+2 x_{1}(t)-x_{2}(t)-x_{3}(t)+x_{4}(t-10)=f_{1}(t), \\
x_{2}^{\prime}(t)-x_{1}(t)+4 x_{2}(t)-x_{3}(t)-x_{4}(t)=f_{2}(t),
\end{gathered}
$$

$$
\begin{aligned}
& x_{3}^{\prime}(t)-x_{1}(t)-x_{2}(t)+4 x_{3}(t)-x_{4}(t)=f_{3}(t), \\
& x_{4}^{\prime}(t)-x_{1}(t)-x_{2}(t)-x_{3}(t)+4 x_{4}(t)=f_{4}(t) .
\end{aligned}
$$

In this case the auxiliary system can be written as follows:

$$
\begin{gathered}
x_{1}^{\prime}(t)+2 x_{1}(t)-x_{2}(t)=z_{1}(t), \\
x_{2}^{\prime}(t)-x_{1}(t)+4 x_{2}(t)-x_{3}(t)-x_{4}(t)=z_{2}(t), \\
x_{3}^{\prime}(t)-x_{1}(t)-x_{2}(t)+4 x_{3}(t)-x_{4}(t)=z_{3}(t), \\
x_{4}^{\prime}(t)-x_{1}(t)-x_{2}(t)-x_{3}(t)+4 x_{4}(t)=z_{4}(t) .
\end{gathered}
$$

We denote by $W(t, s)=\left\{W_{i j}(t, s)\right\}_{i, j=1,2,3,4}$ the Cauchy matrix of this auxiliary system. It can be found that

$$
W(t, s)=\left[\begin{array}{lllll}
\frac{e^{s-t}+e^{3(s-t)}}{2} & \frac{e^{s-t}-e^{5(s-t)}}{4} & \frac{e^{s-t}-2 e^{3(s-t)}+e^{5(s-t)}}{8} & \frac{e^{s-t}-2 e^{3(s-t)}+e^{5(s-t)}}{8} \\
\frac{e^{s-t}-e^{3(s-t)}}{2} & \frac{e^{s-t}+e^{5(s-t)}}{4} & \frac{e^{s-t}+2 e^{3(s-t)}-3 e^{5(s-t)}}{8} & \frac{e^{s-t}+2 e^{3(s-t)}-3 e^{5(s-t)}}{8} \\
\frac{e^{s-t}-e^{3(s-t)}}{2} & \frac{e^{s-t}-e^{5(s-t)}}{4} & \frac{e^{s-t}+2 e^{3(s-t)}+5 e^{5(s-t)}}{8} & \frac{e^{s-t}+2 e^{3(s-t)}-3 e^{5(s-t)}}{8} \\
\frac{e^{s-t}-e^{3(s-t)}}{2} & \frac{e^{s-t}-e^{5(s-t)}}{4} & \frac{e^{s-t}+2 e^{3(s-t)}-3 e^{5(s-t)}}{8} & \frac{e^{s-t}+2 e^{3(s-t)}+5 e^{5(s-t)}}{8}
\end{array}\right] .
$$

Condition (20),

$$
\begin{aligned}
& p_{14} W_{41}(t-10, s)-p_{13} W_{31}(t, s) \\
& \quad=\frac{e^{s-t+10}-e^{3(s-t+10)}-e^{s-t}+e^{3(s-t)}}{2}<0, \\
& p_{14} W_{42}(t-10, s)-p_{13} W_{32}(t, s) \\
& \quad=\frac{e^{s-t+10}-e^{5(s-t+10)}-e^{s-t}+e^{5(s-t)}}{4}<0, \\
& p_{14} W_{43}(t-10, s)-p_{13} W_{33}(t, s) \\
& =\frac{e^{s-t+10}+2 e^{3(s-t+10)}-3 e^{5(s-t+10)}-e^{s-t}-2 e^{3(s-t)}+3 e^{5(s-t)}}{8} \\
& <0,
\end{aligned}
$$

is fulfilled. According to Theorem 3, all elements of the Cauchy matrix $C(t, s)=\left\{C_{i j}(t, s)\right\}_{i, j=1,2,3,4}$ are nonnegative for $0 \leq s \leq t<\infty$. System (37) in this case is of the following form:

$$
\begin{aligned}
& 2 z_{1}-z_{2}-z_{3}+z_{4}=1, \\
& -z_{1}+4 z_{2}-z_{3}-z_{4}=1 \\
& -z_{1}-z_{2}+4 z_{3}-z_{4}=1 \\
& -z_{1}-z_{2}-z_{3}+4 z_{4}=1 .
\end{aligned}
$$

Its solution is $z_{1}=1, z_{2}=1, z_{3}=1$, and $z_{4}=1$, and we get the exponential estimate of the Cauchy matrix $C(t, s)$ of the delay system (52) and the following equalities:

$$
\begin{aligned}
& z_{1}=1=\lim _{t \rightarrow \infty} \sum_{j=1}^{n} \int_{0}^{t} C_{1 j}(t, s) d s, \\
& z_{2}=1=\lim _{t \rightarrow \infty} \sum_{j=1}^{n} \int_{0}^{t} C_{2 j}(t, s) d s, \\
& z_{3}=1=\lim _{t \rightarrow \infty} \sum_{j=1}^{n} \int_{0}^{t} C_{3 j}(t, s) d s, \\
& z_{4}=1=\lim _{t \rightarrow \infty} \sum_{j=1}^{n} \int_{0}^{t} C_{4 j}(t, s) d s .
\end{aligned}
$$

Example 15. The same comments can be noted for the delay system of 5 equations:

$$
\begin{aligned}
& x_{1}^{\prime}(t)+3 x_{1}(t)-x_{2}(t)-x_{3}(t)-x_{4}(t)+x_{5}(t-10) \\
& \quad=f_{1}(t), \\
& x_{2}^{\prime}(t)-x_{1}(t)+5 x_{2}(t)-x_{3}(t)-x_{4}(t)-x_{5}(t)=f_{2}(t), \\
& x_{3}^{\prime}(t)-x_{1}(t)-x_{2}(t)+5 x_{3}(t)-x_{4}(t)-x_{5}(t)=f_{3}(t),
\end{aligned}
$$




$$
\begin{aligned}
& x_{4}^{\prime}(t)-x_{1}(t)-x_{2}(t)-x_{3}(t)+5 x_{4}(t)-x_{3}(t)=f_{4}(t), \\
& x_{5}^{\prime}(t)-x_{1}(t)-x_{2}(t)-x_{3}(t)-x_{4}(t)+5 x_{5}(t)=f_{5}(t) .
\end{aligned}
$$

Auxiliary system (17) can be written as follows:

$$
\begin{gathered}
x_{1}^{\prime}(t)+3 x_{1}(t)-x_{2}(t)-x_{3}(t)=z_{1}(t), \\
x_{2}^{\prime}(t)-x_{1}(t)+5 x_{2}(t)-x_{3}(t)-x_{4}(t)-x_{5}(t)=z_{2}(t),
\end{gathered}
$$

$$
W(t, s)=\left[\begin{array}{cccc}
\frac{e^{s-t}+2 e^{4(s-t)}}{3} & \frac{e^{s-t}-e^{6(s-t)}}{5} & \frac{e^{s-t}-e^{6(s-t)}}{5} \\
\frac{e^{s-t}-e^{4(s-t)}}{3} & \frac{e^{s-t}+4 e^{6(s-t)}}{5} & \frac{e^{s-t}-e^{6(s-t)}}{5} \\
\frac{e^{s-t}-e^{4(s-t)}}{3} & \frac{e^{s-t}-e^{6(s-t)}}{5} & \frac{e^{s-t}+4 e^{6(s-t)}}{5} \\
\frac{e^{s-t}-e^{4(s-t)}}{3} & \frac{e^{s-t}-e^{6(s-t)}}{5} & \frac{e^{s-t}-e^{6(s-t)}}{5} \\
\frac{e^{s-t}-e^{4(s-t)}}{3} & \frac{e^{s-t}-e^{6(s-t)}}{5} & \frac{e^{s-t}-e^{6(s-t)}}{5}
\end{array}\right.
$$

$$
\left.\begin{array}{ll}
\frac{2 e^{s-t}-5 e^{4(s-t)}+3 e^{6(s-t)}}{15} & \frac{2 e^{s-t}-5 e^{4(s-t)}+3 e^{6(s-t)}}{15} \\
\frac{4 e^{s-t}+5 e^{4(s-t)}-9 e^{6(s-t)}}{30} & \frac{4 e^{s-t}+5 e^{4(s-t)}-9 e^{6(s-t)}}{30} \\
\frac{4 e^{s-t}+5 e^{4(s-t)}-9 e^{6(s-t)}}{30} & \frac{4 e^{s-t}+5 e^{4(s-t)}-9 e^{6(s-t)}}{30} \\
\frac{4 e^{s-t}+5 e^{4(s-t)}+21 e^{6(s-t)}}{30} & \frac{4 e^{s-t}+5 e^{4(s-t)}-9 e^{6(s-t)}}{30} \\
\frac{4 e^{s-t}+5 e^{4(s-t)}-9 e^{6(s-t)}}{30} & \frac{4 e^{s-t}+5 e^{4(s-t)}+21 e^{6(s-t)}}{30}
\end{array}\right]
$$

Verifying condition (20), we get

$$
\begin{gathered}
p_{15} W_{51}(t-10, s)-p_{14} W_{41}(t, s) \\
=\frac{e^{s-t+10}-e^{4(s-t+10)}-e^{s-t}+e^{4(s-t)}}{3}<0, \\
p_{15} W_{52}(t-10, s)-p_{14} W_{42}(t, s) \\
=\frac{e^{s-t+10}-e^{6(s-t+10)}-e^{s-t}+e^{6(s-t)}}{5}<0, \\
p_{15} W_{53}(t-10, s)-p_{14} W_{43}(t, s) \\
=\frac{e^{s-t+10}-e^{6(s-t+10)}-e^{s-t}+e^{6(s-t)}}{5}<0, \\
p_{15} W_{54}(t-10, s)-p_{14} W_{44}(t, s) \\
=\left(4 e^{s-t+10}+5 e^{4(s-t+10)}-9 e^{6(s-t+10)}-4 e^{s-t}\right. \\
\left.\quad-5 e^{4(s-t)}-21 e^{6(s-t)}\right)(30)^{-1}<0 .
\end{gathered}
$$

According to Theorem 3, all elements of the Cauchy matrix $C(t, s)=\left\{C_{i j}(t, s)\right\}_{i, j=1,2,3,4,5}$ are nonnegative for $0 \leq$ $s \leq t<\infty$. System (37) in this case is of the following form:

$$
\begin{aligned}
& 3 z_{1}-z_{2}-z_{3}-z_{4}+z_{5}=1, \\
& -z_{1}+5 z_{2}-z_{3}-z_{4}-z_{5}=1, \\
& -z_{1}-z_{2}+5 z_{3}-z_{4}-z_{5}=1, \\
& -z_{1}-z_{2}-z_{3}+5 z_{4}-z_{5}=1, \\
& -z_{1}-z_{2}-z_{3}-z_{4}+5 z_{5}=1 .
\end{aligned}
$$

Its solution is $z_{1}=1, z_{2}=1, z_{3}=1, z_{4}=1$, and $z_{5}=1$, and we get the exponential estimate of the Cauchy matrix $C(t, s)$ of the delay system and also the following equalities:

$$
\begin{aligned}
& z_{1}=1=\lim _{t \rightarrow \infty} \sum_{j=1}^{n} \int_{0}^{t} C_{1 j}(t, s) d s, \\
& z_{2}=1=\lim _{t \rightarrow \infty} \sum_{j=1}^{n} \int_{0}^{t} C_{2 j}(t, s) d s, \\
& z_{3}=1=\lim _{t \rightarrow \infty} \sum_{j=1}^{n} \int_{0}^{t} C_{3 j}(t, s) d s, \\
& z_{4}=1=\lim _{t \rightarrow \infty} \sum_{j=1}^{n} \int_{0}^{t} C_{4 j}(t, s) d s, \\
& z_{5}=1=\lim _{t \rightarrow \infty} \sum_{j=1}^{n} \int_{0}^{t} C_{5 j}(t, s) d s .
\end{aligned}
$$

\section{Conflict of Interests}

The authors declare that there is no conflict of interests regarding the publication of this paper.

\section{Acknowledgments}

This paper appeared as a result of theoretical discussions around the project "Exploitation of a Synergetic Model for Development of Business of Innovation Type" on the Topic no. 2013/276-C "Development of a Model of Operation of Innovation Business as Dynamic Model with Memory Effect," 
supported by Perm National Research Polytechnic University with financial support of Ministry of Science and Education of Russian Federation (Agreement no. 02.G25.31.0068 of 23.05.2013).

\section{References}

[1] S. A. Tchaplygin, New Method of Approximate Integration of Differential Equations, GTTI, Moscow, Russia, 1932.

[2] N. N. Luzin, "About method of approximate integration of acad. S. A. Chaplygin," Uspehi Mathematicheskih Nauk, vol. 6, no. 6, pp. 3-27, 1951.

[3] V. Lakshmikantham and S. Leela, Differential and Integral Inequalities, Academic Press, 1969.

[4] I. Kiguradze and B. Puza, Boundary Value Problems for Systems of Linear Functional Differential Equations, FOLIA, Brno, Czech Republi, 2002.

[5] N. V. Azbelev, V. P. Maksimov, and L. F. Rakhmatullina, Introduction to the Theory of Functional Differential Equations, Advanced Series in Math. Science and Engineering 3, World Federation, Atlanta, Ga, USA, 1995.

[6] T. Wazewsk, "Systemes des equations et des inegalites differentielled aux deuxieme membres et leurs applications," Annales Polonici Mathematici, vol. 23, pp. 112-166, 1950.

[7] A. I. Domoshnitsky and M. V. Sheina, "Nonnegativity of the Cauchy matrix and the stability of a system of linear differential equations with retarded argument," Differentsial'nye Uravnenija, vol. 25, no. 2, pp. 201-208, 1989.

[8] I. Kiguradze and B. Puza, "On boundary value problems for systems of linear functional differential equations," Czechoslovak Mathematical Journal, vol. 47, no. 2, pp. 341-373, 1997.

[9] I. Kiguradze, "Boundary value problems for systems of ordinary differential equations," Itogi Nauki Tech., Ser. Sovrem. Probl. Mat., Novejshie Dostizh., vol. 30, pp. 3-103, 1987 (Russian), English transl.: Journal of Soviet Mathematics, vol. 43, no. 2, pp. 2259-2339, 1988.

[10] D. Bainov and A. Domoshnitsky, "Nonnegativity of the Cauchy matrix and exponential stability of a neutral type system of functional-differential equations," Extracta Mathematicae, vol. 8, no. 1, pp. 75-82, 1993.

[11] I. Gyori, "Interaction between oscillation and global asymptotic stability in delay differential equations," Differential \& Integral Equations, vol. 3, pp. 181-200, 1990.

[12] S. A. Campbell, "Delay independent stability for additive neural networks," Differential Equations and Dynamical Systems, vol. 9, no. 3-4, pp. 115-138, 2001.

[13] J. Hofbauer and J. W.-H. So, "Diagonal dominance and harmless off-diagonal delays," Proceedings of the American Mathematical Society, vol. 128, no. 9, pp. 2675-2682, 2000.

[14] R. P. Agarwal, L. Berezansky, E. Braverman, and A. Domoshnitsky, Nonoscillation Theory of Functional Differential Equations with Applications, Springer, New York, NY, USA, 2012.

[15] I. Gyori and F. Hartung, "Fundamental solution and asymptotic stability of linear delay differential equations," Dynamics of Continuous, Discrete and Impulsive Systems, vol. 13, no. 2, pp. 261-287, 2006.

[16] A. Domoshnitsky, "About applicability of Chaplygin's theorem to one component of the solution vector," Differentsial'nye Uravnenija, vol. 26, no. 10, pp. 1699-1705, 1990.

[17] R. P. Agarwal and A. Domoshnitsky, "On positivity of several components of solution vector for systems of linear functional differential equations," Glasgow Mathematical Journal, vol. 52, no. 1, pp. 115-136, 2010.

[18] A. Domoshnitsky, "About maximum principles for one of the components of solution vector and stability for systems of linear delay differential equations," in Proceedings of the 8th AIMS Conference on Discrete and Continuous Dynamical Systems, pp. 373-380, American Institute of Mathematical Sciences, Dresden, Germany, 2011.

[19] L. Berezansky, E. Braverman, and A. Domoshnitsky, "On nonoscillation of systems of delay equations," Funkcialaj Ekvacioj, vol. 54, no. 2, pp. 275-296, 2011.

[20] A. Domoshnitsky, "Maximum principles and nonoscillation intervals for first order Volterra functional differential equations," Dynamics of Continuous, Discrete and Impulsive Systems A: Mathematical Analysis, vol. 15, no. 6, pp. 769-814, 2008. 


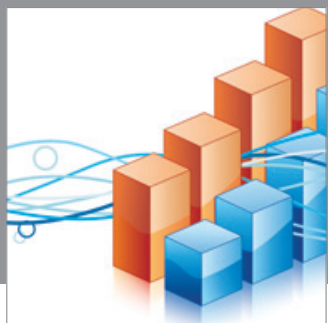

Advances in

Operations Research

mansans

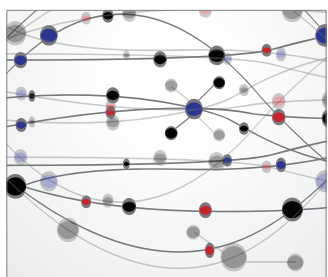

The Scientific World Journal
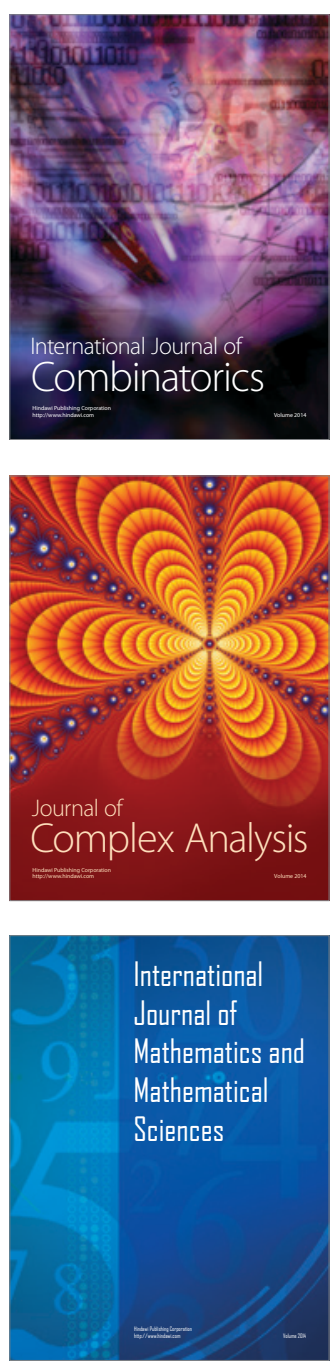
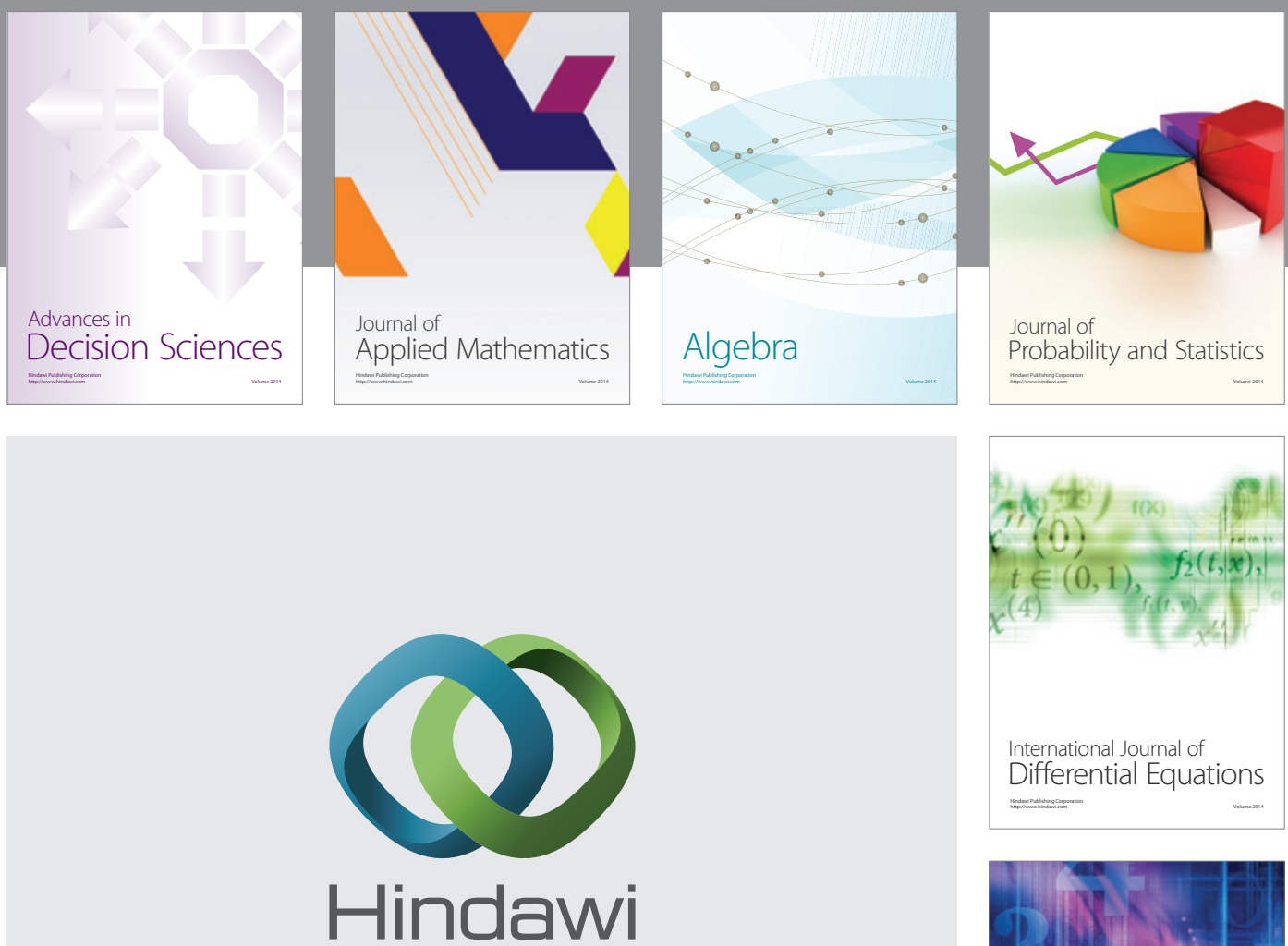

Submit your manuscripts at http://www.hindawi.com
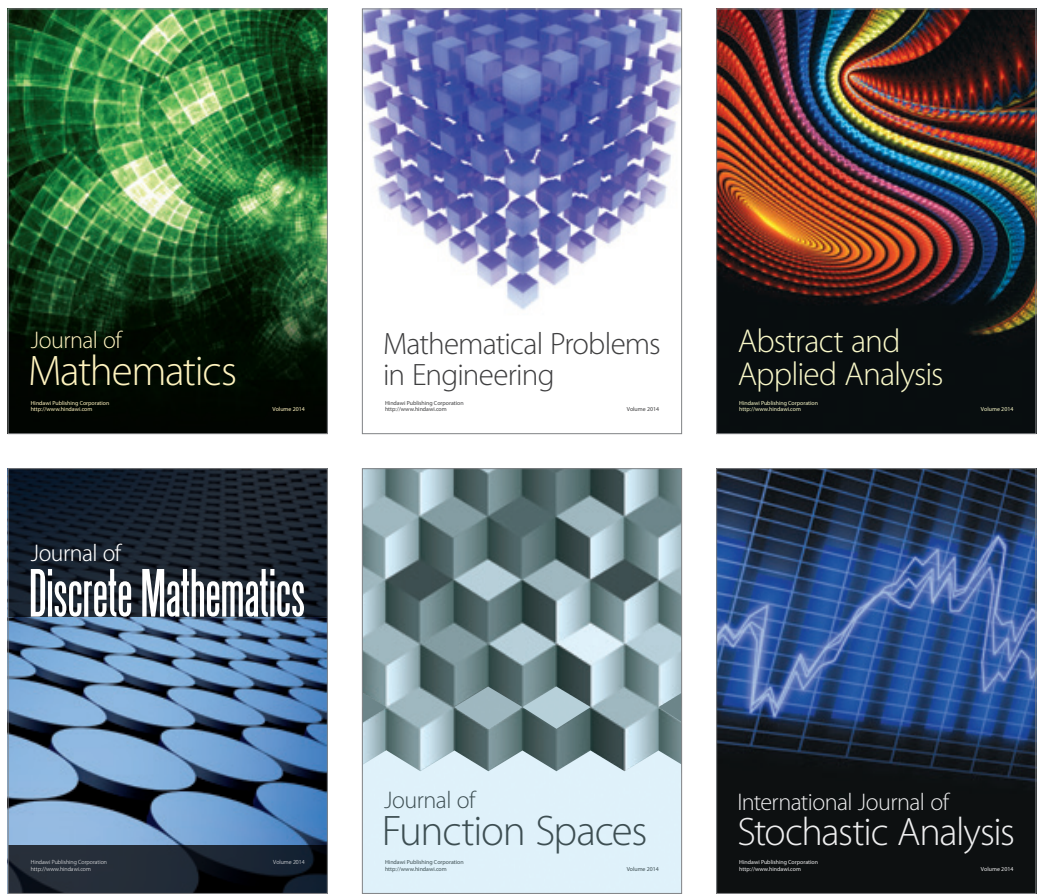

Journal of

Function Spaces

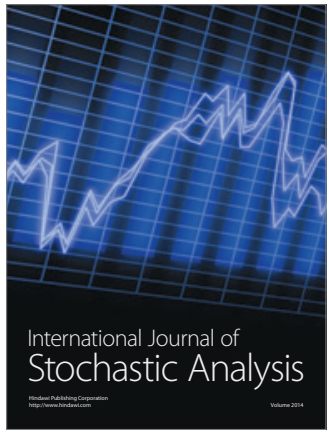

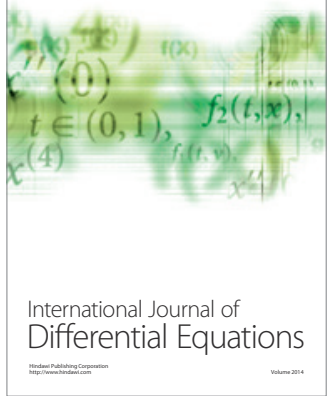
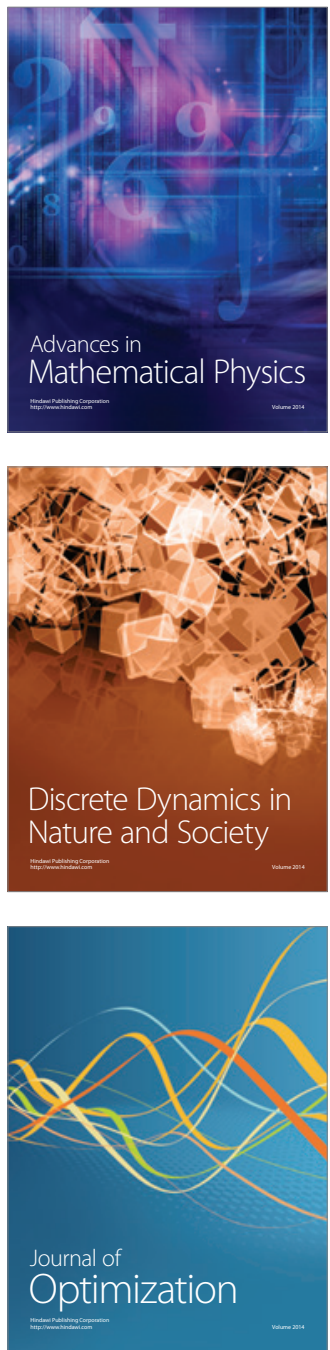